\title{
Time Quanta Present in Simple Quantum Systems
}

\author{
Stanisław Olszewski \\ Institute of Physical Chemistry, Polish Academy of Sciences, Warsaw, Poland \\ Email: olsz@ichf.edu.pl
}

How to cite this paper: Olszewski, S. (2021) Time Quanta Present in Simple Quantum Systems. Journal of Modern Physics, 12, 1005-1011.

https://doi.org/10.4236/jmp.2021.127062

Received: April 9, 2021

Accepted: May 22, 2021

Published: May 25, 2021

Copyright (c) 2021 by author(s) and Scientific Research Publishing Inc. This work is licensed under the Creative Commons Attribution International License (CC BY 4.0).

http://creativecommons.org/licenses/by/4.0/ (c) (i) Open Access

\begin{abstract}
Differences of the time periods in two independent quantum systems are examined on a semiclassical level. The systems are the electron in the hydrogen atom and a free-electron particle moving in a one-dimensional potential box, respectively. It is demonstrated that in both systems the relativistic correction to the time interval can be expressed as a multiple of the same quantum of time. The size of the quantum is proportional to the ratio of the Planck's constant and the rest energy of the electron particle.
\end{abstract}

\section{Keywords}

Time Periods of the Electron Oscillations in the Bohr Hydrogen Atom and in a One-Dimensional Potential Box, Relativistic Correction to the Difference of the Time Periods, Time Quanta

\section{Introduction}

The problem of the time calculation seldom discussed in the quantum theory can remain actual even for very simple quantum systems. These systems are often of the periodic character, so we need the time periods of the electron motion as parameters. This kind of parameter is usually approached in a semiclassical way. Perhaps the best known example concerns the time periods of the electron orbital motion in the hydrogen atom [1] [2].

Other well-known examples of a semiclassical motion are the free particle in a potential box and the harmonic oscillator. In the last case a difficulty concerning time is provided by an unstable size of the velocity connected with a moving particle.

In order to make the problem of calculations of the time periods to be very simple, only the electron in the hydrogen atom and the particle moving in a 
one-dimenssional potential box are considered exactly in the paper. The time quanta in the harmonic oscillator are calculated only in an approximate way.

The main aim of the paper was to point out that a relativistic change of a difference between two oscillation periods of time in a quantum system can be obtained with the aid of the time quantum which has its size independent of the examined system.

\section{Electron Circulating in the Hydrogen Atom and Its Time Periods}

A successful Bohr theory of the atom (see e.g. [1]) assumed planar circular orbits about the nucleus. The radius of the $n$th orbit is defined by formula [2]

$$
r_{n}=\frac{n^{2} \hbar^{2}}{m e^{2}}
$$

and the quantum numbers of the orbits are labelled by

$$
n=1,2,3, \cdots, \infty \text {. }
$$

$m$ is the electron mass, $-e$ - electron charge. The electron velocity on the $n$th orbit is [2]

$$
v_{n}=\frac{e^{2}}{n \hbar},
$$

so the time period $T_{n}$ connected with the electron circulating along the same orbit is deduced from the formula

$$
v_{n}=\frac{2 \pi r_{n}}{T_{n}}
$$

giving

$$
T_{n}=\frac{2 \pi r_{n}}{v_{n}}=\frac{2 \pi n^{2} \hbar^{2}}{m e^{2}} \frac{n \hbar}{e^{2}}=\frac{2 \pi n^{3} \hbar^{3}}{m e^{4}} .
$$

The task is to calculate the difference

$$
T_{n_{2}}-T_{n_{1}}
$$

transformed into a relativistic interval of time (see Sec. 3) for an arbitrary pair of the quantum states, say $n_{2}$ and $n_{1}$. Let us assume that

$$
n_{2}>n_{1} \text {, }
$$

so the difference in (6) is a positive number.

\section{Relativistic Difference of the Time Periods Given in (6)}

In order to improve (6) we apply the relativistic theory in the sense that the components entering (6) should be multiplied respectively by the factors (see e.g. [3] [4])

$$
\sqrt{1-\frac{v_{n_{2}}^{2}}{c^{2}}} \approx 1-\frac{1}{2} \frac{v_{n_{2}}^{2}}{c^{2}},
$$




$$
\sqrt{1-\frac{v_{n_{1}}^{2}}{c^{2}}} \approx 1-\frac{1}{2} \frac{v_{n_{1}}^{2}}{c^{2}}
$$

In effect we get a proper relativistic change of the time periods connected with the difference (6). Two terms in each of the power expansions (7) and (8) seem to be sufficient because

$$
v_{n_{2}} \ll c
$$

and

$$
v_{n_{1}} \ll c
$$

We obtain:

$$
T_{n_{2}}^{\text {rel }}-T_{n_{1}}^{\text {rel }}=\sqrt{1-\frac{v_{n_{2}}^{2}}{c^{2}}} T_{n_{2}}-\sqrt{1-\frac{v_{n_{1}}^{2}}{c^{2}}} T_{n_{1}} \cong T_{n_{2}}-T_{n_{1}}-\frac{1}{2 c^{2}}\left(v_{n_{2}}^{2} T_{n_{2}}-v_{n_{1}}^{2} T_{n_{1}}\right) .
$$

Therefore

$$
\begin{aligned}
T_{n_{2}}^{\text {rel }}-T_{n_{1}}^{\text {rel }} & \cong T_{n_{2}}-T_{n_{1}}-\frac{1}{2 c^{2}}\left(\frac{e^{4}}{n_{2}^{2} \hbar^{2}} \frac{2 \pi n_{2}^{3} \hbar^{3}}{m e^{4}}-\frac{e^{4}}{n_{1}^{2} \hbar^{2}} \frac{2 \pi n_{1}^{3} \hbar^{3}}{m e^{4}}\right) \\
& =\frac{2 \pi \hbar^{3}}{m e^{4}}\left(n_{2}^{3}-n_{1}^{3}\right)-\frac{2 \pi \hbar}{2 m c^{2}}\left(n_{2}-n_{1}\right) \\
& =T_{n_{2}}-T_{n_{1}}-\frac{h}{2 m c^{2}}\left(n_{2}-n_{1}\right) .
\end{aligned}
$$

At the end of (12) appeared a supplement having a dimension of time and being proportional to the difference

$$
n_{2}-n_{1}
$$

Also we have that

$$
T_{n_{2}}-T_{n_{1}} \sim n_{2}-n_{1}
$$

because

$$
\left(n_{2}-n_{1}\right)\left(n_{2}^{2}+n_{2} n_{1}+n_{1}^{2}\right)=n_{2}^{3}-n_{1} n_{2}^{2}+n_{2}^{2} n_{1}-n_{2} n_{1}^{2}+n_{2} n_{1}^{2}-n_{1}^{3}=n_{2}^{3}-n_{1}^{3} .
$$

These results make (6) proportional to both (13) and (15).

\section{Electron Oscillation in a One-Dimensional Potential Box and Its Properties}

For a constant potential within the potential box of length $L$ we have the electron energy states [5]

$$
E_{n}=\frac{n^{2} h^{2}}{8 m L^{2}}
$$

where $n$ is the integer quantum number, $m$ the electron mass, and $h$ is the Planck constant.

Formula (16) is equivalent to the kinetic energy expression

$$
E_{n}=\frac{m}{2} v_{n}^{2},
$$


therefore by putting (16) equal to (17) we obtain the electron velocity in the potential box:

$$
v_{n}=\frac{n h}{2 m L} .
$$

For a given $n$ this velocity can be considered as having a constant value for the whole length $L$.

Due to the boundary conditions at the box ends, the electron behaves like a free particle oscillating along the length

$$
L+L=2 L
$$

for each oscillation time period $T_{n}$. A non-relativistic approach to $T_{n}$ gives from (18):

$$
T_{n}=\frac{2 L}{v_{n}}=\frac{4 m L^{2}}{n h} .
$$

Our aim is to examine the difference

$$
T_{n_{2}}-T_{n_{1}} .
$$

The assumption of $n_{2}>n_{1}$ implies a negative time difference in (21); see (20).

\section{A Relativistic Difference of the Time Periods Entering (21)}

A constant velocity (18) implies an approximately free-electron motion along the distance $L$ entering (19). A relativistic modification of the time difference (21) becomes similar to that obtained in the case of the difference (6):

$$
T_{n_{2}}^{\mathrm{rel}}-T_{n_{1}}^{\mathrm{rel}}=\sqrt{1-\frac{v_{n_{2}}^{2}}{c^{2}}} T_{n_{2}}-\sqrt{1-\frac{v_{n_{1}}^{2}}{c^{2}}} T_{n_{1}} \cong T_{n_{2}}-T_{n_{1}}-\frac{1}{2 c^{2}}\left(v_{n_{2}}^{2} T_{n_{2}}-v_{n_{1}}^{2} T_{n_{1}}\right),
$$

where the term in brackets in (22) is a positive number; see (24). We have

$$
T_{n_{2}}-T_{n_{1}}=\frac{4 m L^{2}}{h}\left(\frac{1}{n_{2}}-\frac{1}{n_{1}}\right)=\frac{4 m L^{2}}{h} \frac{n_{1}-n_{2}}{n_{1} n_{2}}<0
$$

because of the assumption done below (21). But in the next step:

$$
\begin{aligned}
\frac{1}{2 c^{2}}\left(v_{n_{2}}^{2} T_{n_{2}}-v_{n_{1}}^{2} T_{n_{1}}\right) & =\frac{1}{2 c^{2}}\left[\left(\frac{n_{2} h}{2 m L}\right)^{2} \frac{4 m L^{2}}{n_{2} h}-\left(\frac{n_{1} h}{2 m L}\right)^{2} \frac{4 m L^{2}}{n_{1} h}\right] \\
& =\frac{1}{2 c^{2}}\left(\frac{n_{2} h}{m}-\frac{n_{1} h}{m}\right)=\frac{h}{2 m c^{2}}\left(n_{2}-n_{1}\right)>0 .
\end{aligned}
$$

A sum of results obtained in (23) and (24) (taken with a minus sign) gives

$$
T_{n_{2}}^{\text {rel }}-T_{n_{1}}^{\text {rel }}=T_{n_{2}}-T_{n_{1}}-\frac{h}{2 m c^{2}}\left(n_{2}-n_{1}\right)
$$

which is formally equal to the result calculated in (12). Evidently see (23) the difference

$$
T_{n_{2}}-T_{n_{1}}
$$


is proportional to $n_{1}-n_{2}$. The component (24)-because of its minus sign in (22) makes the whole expression (25) to get the same property of proportionality.

A supplement obtained at the end of (25) is identical to that entering (12) calculated for the hydrogen atom. This enables us to consider the term having

$$
\frac{h}{2 m c^{2}}
$$

as a quantum of time correcting the difference of the time periods

$$
T_{n_{2}}-T_{n_{1}}
$$

obtained either for the hydrogen atom, or in the potential box.

\section{Approximate Approach to the Time Quanta in Case of the Harmonic Oscillator}

In this case we assume-for the sake of simplicity-that the time periods $T$ belonging to different quantum states are equal giving the circular frequency

$$
\omega=\frac{2 \pi}{T}=\sqrt{\frac{k}{m}}
$$

where $k$ is the oscillation constant and $m$ is the mass of the oscillator [6]. All energy states are given by the formula

$$
E_{n}=E_{m}^{(\mathrm{kin})}+E_{n}^{(\mathrm{pot})}=\frac{k a_{n}^{2}}{2}
$$

where $a_{n}$ is the oscillator amplitude characteristic for state $n$.

The states are equally separated by the terms $\hbar \omega$ :

$$
E_{n}=n \hbar \omega=\frac{k a_{n}^{2}}{2} .
$$

The velocity $v_{n}$ of the oscillating particle between the limits

$$
-a_{n}<x<a_{n}
$$

has its maximum at a central particle position $x=0$.

In calculating the relativistic correction to the time period $T$ we take into account-for the sake of simplicity—the average particle velocity

$$
\bar{v}_{n}=\frac{4 a_{n}}{T} .
$$

In effect the relativistic correction to $T$ becomes:

$$
T_{n}^{\text {rel }} \cong \sqrt{1-\frac{\bar{v}_{n}^{2}}{c^{2}}} T \cong \sqrt{1-\left(\frac{4 a_{n}}{T}\right)^{2} \frac{1}{c^{2}}} T \cong\left[1-\frac{1}{2}\left(\frac{4 a_{n}}{T}\right)^{2} \frac{1}{c^{2}}\right] T .
$$

But because of (30) we have

$$
a_{n}^{2}=\frac{2 E_{n}}{k}
$$

which gives with the aid of the formula (28) for $\omega$ 


$$
\begin{aligned}
T_{n}^{\mathrm{rel}}-T & =-\frac{1}{2} \frac{\left(4 a_{n}\right)^{2}}{T c^{2}}=-\frac{16 E_{n}}{k T c^{2}}=-\frac{16 n \hbar \omega}{k T c^{2}} \\
& =-\frac{8}{\pi} \frac{n \hbar \omega^{2}}{k c^{2}}=-\frac{8}{\pi} \frac{n \hbar}{m c^{2}}=-\frac{8}{\pi^{2}} \frac{n h}{2 m c^{2}} .
\end{aligned}
$$

\section{Summary}

We examined the differences of the time periods possessed by the electron either in the case of its circulation in the hydrogen atom, or when the electron is enclosed as a particle moving in a one-dimensional potential box. The approach to the time properties of each of these physical systems is modified by the relativistic theory.

It is found that contribution given by the relativistic term of time for both systems is the same. This enables us to consider the relativistic corrections introduced to the differences of the time periods as representing the quanta of time: the size of quanta is proportional to the difference of the quantum numbers which define separately any of the time periods present in each system. Therefore, in order to obtain the correction, the quantum numbers multiply solely one term independent of the physics of the examined system. This term is a constant ratio

$$
\frac{h}{2 m c^{2}} \cong 0.4 \times 10^{-20} \mathrm{sec}
$$

built up of the fundamental constants of nature. It can be noted that the denominator entering the fraction in (36) is equal to the Dirac's energy expression separating theseas of electron particles and antiparticles; see e.g. [7].

An approximate treatment concerning (36) was applied in calculating the time quanta of the harmonic oscillator. In this case the non-relativistic oscillation periods of time are assumed to be equal for all quantum levels and the relativistic correction concerns-in average-solely an individual oscillation period of time. In effect of the used approximations the result in (36) should be multiplied by a constant factor

$$
\frac{8}{\pi^{2}}
$$

which is not much different than unity.

\section{Acknowledgements}

The paper is elaborated in course of my prayers to the Almighty God for a young Victoria-suddenly deceased.

\section{Conflicts of Interest}

The author declares no conflicts of interest regarding the publication of this paper.

\section{References}

[1] Bohr, N. (1992) The Theory of Spectra and the Atomic Constitution. Cambridge 
University Press, Cambridge, UK.

[2] Sommerfeld, A. (1931) Atombau und Spektrallinien. Vol. 1, 5th Edition, Vieweg, Braunschweig.

[3] Jauncey, G.E.M. (1948) Modern Physics. 3rd Edition, Van Nostrand, New York.

[4] Landau, L.D. and Lifshits, E.M. (1969) Mechanics. Electrodynamics. Izd. Nauka, Moscow (in Russian).

[5] Eyring, H., Walter, J. and Kimball, G.E. (1957) Quantum Chemistry. Wiley, New York.

[6] Sommerfeld, A. (1949) Mechanik. Akademische Verlagsgesellschaft, Leipzig.

[7] Slater, J.C. (1967) Quantum Theory of Molecules and Solids. Vol. 3, McGraw Hill, New York. 